\title{
Local and global stability analysis for Gilpin-Ayala competition model involved in harmful species via LMI approach and variational methods
}

\author{
Ruofeng Rao ${ }^{\mathrm{a}}$ \\ ${ }^{a}$ Department of Mathematics, Chengdu Normal University, Chengdu , 611130, China
}

\begin{abstract}
Firstly, the author do dynamic analysis for reaction-diffusion Gilpin-Ayala competition model with Dirichlet boundary value, involved in harmful species. Existence of multiple stationary solutions is verified by way of Mountain Pass lemma, and the local stability result of the null solution is obtained by employing linear approximation principle. Secondly, the author utilize variational methods and LMI technique to deduce the LMI-based global exponential stability criterion on the null solution which becomes the unique stationary solution of the ecosystem with delayed feedback under a reasonable boundedness assumption on population densities. Particularly, LMI criterion is involved in free weight coefficient matrix, which reduces the conservatism of the algorithm. In addition, a new impulse control stabilization criterion is also derived. Finally, two numerical examples show the effectiveness of the proposed methods. It is worth mentioning that the obtained stability criteria of null solution presented some useful hints on how to eliminate pests and bacteria.
\end{abstract}

Keywords: Gilpin-Ayala competition model; linear matrix inequality (LMI) ; Mountain Pass lemma; variational methods; Markovian jumping ; impulse control on stabilization

\section{Introduction}

In 1920, Lotka and Volterra originally proposed the famous population competition model ([1,2]), which is recognized and cited by many scholars ([3-9]). Because of the diffusion of population in space, some reaction-diffusion models were investigated in order to better simulate the real ecological situation $([3,4]$ and their references therein). Besides, practice has verified that nonlinear models are often able to better simulate the actual situation. In 1973, Gilpin and Ayala found that the linear competition model was not consistent with the experimental results ([5]). Through accurate data analysis, they proposed a nonlinear competition model of two populations, called as GilpinAyala competition model (GACM) by adding the nonlinear density constraint parameters. As pointed out in [6-9], when each of nonlinear density constraint parameters is much less than 1, GACM can well simulate the population ecology of Drosophila melanogaster. Naturally, reaction-diffusion Gilpin-Ayala models had been studied (see, e.g. [20, 24]). But most of the related literatures mainly focus on the study of the competition model under Neumann boundary

Email address: ruofengrao@163.com; ruofengrao@cdnu.edu.cn (Ruofeng Rao )

Preprint submitted to $* * *$

February 24, 2021 
value condition ([3,4,8] and related references), and the diffusive ecosystem under Dirichlet zero-boundary value is rarely studied. In fact, the Drichlet boundary value diffusion ecosystem can better reflect the actual situation for a class of species that can not live on the edge of the biosphere, for example, rabbits don't live on the edge of the grass where the degree of desertification is very serious. Recently, Ruofeng Rao, Quanxin Zhu and Kaibo Shi investigated the stability of positive stationary solution of reaction-diffusion Gilpin-Ayala competition model (RDGACM), but the null solution of RDGACM is not studied still. In fact, if we wish that pest or bacterial populations are destroyed, the stability of the null solution should be investigated. This inspires us to write this paper.

In this paper, our paper has the following innovative points:

(I1) This paper, the author consider the stability of the null solution of RDGACM. Seldom papers involved in the stability of the null solution of ecosystem([1-9,20,24]), but it has to be studied due to some practical situation. For example, rabbits are rampant in Australia, and carp are rampant in the United States. The stability analysis of the zero solution of this paper is closely related to the extinction of harmful species.

(I2) RDGACM with Dirichlet zero-boundary value is considered in this paper, which is suitable to research on a class of species that can not live on the edge of the biosphere. But there are many literature only involved in Neumann zero-boundary value([3,4,8]). Seldom papers involved in Dirichlet zero-boundary value.

(I3) Dirichlet zero-boundary value of this paper brings about mathematical difficulties in the dynamical analysis. According [11, Remark 9], non-zero constant function may be a stationary solution of reaction-diffusion system with Neumann zero-boundary value, which can always be obtained by solving a simple algebraic equations. But [11, statement 1] shows that non-zero constant function must be not a stationary solution in the case of Dirichlet zero-boundary value. So in this paper we have to use variational methods to prove the existence or unique existence of stationary solution in the case of Dirichlet zero-boundary value. This is a main difficulty different from previous literature related to dynamical system with Neumann zero-boundary value $([3,4,8,25,26]$ and their references therein). In fact, except $[25,26]$ involving more complex mathematical tools, such as Laplacian semigroup or variational methods, most of the literature related with Neumann zero-boundary value only involved in simple mathematical methods dealing with the unique existence of the equilibrium point. In this paper, on one hand, the author utilize Mountain Pass Lemma to derive the existence of multiple stationary solutions. On the other hand, under boundedness assumption on the population densities, variational technique is applied to deal with the uniqueness of the stationary solution.

(I4) LMI-based criterion and free weight coefficient matrix technique reduce the conservatism of the algorithm in this paper. In fact, we seldom find out the LMI-based criterion in previous literature involved in nonlinear ecosystem (see. e.g., [1-9,20] and their references therein). Although LMI-based criterion or free weight coefficient matrix technique is common in many literature related to reaction-diffusion nonlinear system, such as [23], but the nonlinear active function of [23] is globally Lipschitz continuous, and the "active function" of this paper is locally Lipschitz continuous.

(I5) Markovian jumping model is introduced in this paper, different from many related literature ([1-9]). Delayed 
feedback is because of the fact that only adults compete in the model, and there is a growth period from larva to adult. Particularly, the growth period is always affected by weather, temperature, humidity and other random factors, which can be reduced to a limited number of modes by specific statistical data.

(I6) In this paper, we shall give the sufficient condition on the existence of multiple stationary solutions of GACM, which directly warrants the local stability of the zero solution. This shows vividly the perfection of the sufficient condition.

In next sections, we shall give some models description in the chapter 2, and dynamic analysis for the GilpinAyala competition model in Section 3, including the existence of multiple equilibrium points, and particularly caring about stability of the zero solution. In Chapter 4, the author will care about the global stability of the zero solution of RDGACM, which should be the unique equilibrium point under some suitable assumptions. Next, two numerical examples will be presented to show the effectiveness of the obtained results. Finally, some interesting conclusions will be proposed in Section 6.

For convenience, we introduce the following notations in this paper:

- The inequality $A>B$ or $B<A$ represents $(A-B)$ is a positive definite matrix for two symmetric matrices $A$ and $B$; - Denote by $\lambda_{1}$ the first positive eigenvalue of the Laplace operator $-\Delta$ in $H_{0}^{1}(\Omega)$, and by $\|u\|=\sqrt{\int_{\Omega}|\nabla u(x)|^{2} d x}$ the norm of Sobolev space $H_{0}^{1}(\Omega)$;

○ Denote simply $\tau_{r}(t)=\tau(r(t), t)$ for $r(t)=r \in S$, where $S=\left\{1,2, \cdots, n_{0}\right\}$;

○ Denote simply $\dot{\tau}_{r}(t)=\frac{d}{d t} \tau(r(t), t)$ for $r(t)=r \in S$, where $S=\left\{1,2, \cdots, n_{0}\right\}$;

- The mark $*$ in a symmetric matrix represents the symmetric terms in the symmetric matrix;

$\circ$ Denote $\mathbb{Z}^{+}=\{1,2, \cdots\}$, and $t_{0}=0$;

○ Denote $A^{-1}$ for any invertible matrix $A$;

- Denote by $\lambda_{\max } A$ and $\lambda_{\min } A$ the maximum eigenvalue and minimum eigenvalue of a symmetric matrix $A$, respectively.

\section{Preliminaries}

Consider the nonlinear Reaction-diffusion Gilpin-Ayala competition model (RDGACM) under Dirichlet boundary value:

$$
\left\{\begin{array}{l}
\frac{\partial u_{1}}{\partial t}=d_{1} \Delta u_{1}+u_{1}\left(b_{1}-a_{11} u_{1}^{\theta_{1}}-a_{12} u_{2}\right), \quad t \geqslant 0, x \in \Omega, \\
\frac{\partial u_{2}}{\partial t}=d_{2} \Delta u_{2}+u_{2}\left(b_{2}-a_{21} u_{1}-a_{22} u_{2}^{\theta_{2}}\right), \quad t \geqslant 0, x \in \Omega, \\
u_{1}(t, x)=u_{2}(t, x)=0, \quad t \geqslant 0, x \in \partial \Omega, \\
u_{1}(0, x)=\xi_{1}(x), \quad u_{2}(0, x)=\xi_{2}(x),
\end{array}\right.
$$

where $\Omega$ is a domain in $\mathbb{R}^{n}(n \in\{2,3\})$ with the smooth boundary $\partial \Omega$. For $i=1,2, u_{i}(t, x)$ represents the population density of the $i$ th population at time $t$ and the spatial location $x, b_{i}>0$ represents the birth rate of the population of the $i$ th species, and $a_{i j}>0$ represents the competition parameter between the species $i$ and the species $j . d_{i}>0$ represents the diffusion coefficient for the species $i$. Initial value function $\xi_{i}(x)$ is bounded and continuous. 
Remark 1. Note $u_{1}(t, x)=u_{2}(t, x)=0, t \geqslant 0, x \in \partial \Omega$, it is the so-called Dirichlet zero-boundary value. Based on the viewpoint (I3) in the introduction section, the RDGACM (2.1) well simulates the actual situation of a class of species. Of course, this model (2.1) brings about some mathematical difficulties in dynamical analysis, and so we need the following lemma, known as Mountain Pass Lemma ([12]):

Lemma 2.1 (Mountain Pass Lemma without the (PS) condition). Let $X$ is a Banach space, $\Psi \in C^{1}(X, \mathbb{R})$, satisfying $\Psi(0)=0$, and there exists $\rho>0$ such that $\left.\Psi\right|_{\partial B_{\rho}(0)} \geqslant \alpha>0$. Besides, there is $e \in X \backslash \overline{B_{\rho}(0)}$ such that $\Psi(e) \leqslant 0$. Let $\Gamma$ be the set of all paths connecting 0 and $e$. That is,

$$
\Gamma=\left\{\psi \in C\left([0,1], H_{0}^{1}(\Omega)\right): \psi(0)=0, \psi(1)=e\right\}
$$

Set

$$
c_{*}=\inf _{\psi \in \Gamma} \max _{s \in[0,1]} \Psi(\psi(s)) .
$$

Then $c_{*} \geqslant \alpha$, and $\Psi$ possesses a critical sequence on $c_{*}$.

Remark 2. Lemma 2.1 is the so-called Mountain Pass Lemma ([12, 21]) without the (PS) condition, and the so-called (PS) condition can be found in many literature (see, e.g. $[11,12,21]$ ). If, in addition, $\Psi$ satisfies the (PS) condition, then $c_{*}$ is a critical value of $\Psi$.

As pointed out in [6-9], when the parameter $\theta_{i}$ is much less than 1 , the nonlinear density constrained model can well simulate the population ecology of Drosophila melanogaster. So we assume $\theta_{i} \in(0,1)$, and consider the following condition for the upcoming section 3 :

(H1) For each $i \in\{1,2\}$, there are positive numbers $p_{i}, q_{i}$ such that $\frac{p_{i}}{q_{i}}-2=\theta_{i} \in(0,1)$, where $p_{i}$ and $q_{i}$ are a pair of Coprime odd numbers.

Below, we may firstly give a dynamical analysis for GACM (2.1) in the section 3. After giving it, we shall further study the stability of delayed feedback Markovian jumping RDGACM in view of the viewpoint (I5) in the introduction section.

\section{Dynamical analysis for RDGACM}

In this section, we firstly analyze the number of equilibrium points on the phase plane of the RDGACM (2.1) with Dirichlet zero-boundary value.

Theorem 3.1. Suppose (H1) holds, $b_{i}<d_{i} \lambda_{1}$ and $0<\theta_{i}<1, \forall i=1,2$. Then the RDGACM (2.1) possesses at least three stationary solutions $(0,0),\left(u_{1 *}(x), 0\right)$ and $\left(0, u_{2 *}(x)\right)$, where $u_{i *}(x) \neq 0, \forall i=1,2$.

Proof. Due to $\Omega \subset \mathbb{R}^{2}$ or $\Omega \subset \mathbb{R}^{3}$, we may consider the case of $\Omega \subset \mathbb{R}^{3}$, and another case can be similarly proved.

Firstly, $(0,0)$ is a trivial solution of the system $(2.1)$.

Next, if $\left(u_{1}(x), 0\right)$ is a stationary solution of the system $(2.1)$,

$$
\left\{\begin{array}{l}
d_{1} \Delta u_{1}(x)+b_{1} u_{1}(x)-a_{11} u_{1}(x)^{1+\theta_{1}}=0, \quad \text { a.e. } x \in \Omega, \\
u_{1}(x)=0, \quad x \in \partial \Omega .
\end{array}\right.
$$

Similarly, if $\left(0, u_{2}(x)\right)$ is a stationary solution of the system (2.1),

$$
\left\{\begin{array}{l}
d_{2} \Delta u_{2}(x)+b_{2} u_{2}(x)-a_{22} u_{2}(x)^{1+\theta_{2}}=0, \\
u_{2}(x)=0, \quad x \in \partial \Omega .
\end{array}\right.
$$


Obviously,

$$
J\left(u_{1}\right)=\frac{1}{2} d_{1}\left\|u_{1}\right\|^{2}-\frac{1}{2} b_{1} \int_{\Omega} u_{1}^{2} d x+\frac{a_{11}}{2+\theta_{1}} \int_{\Omega} u_{1}^{2+\theta_{1}} d x
$$

is the functional corresponding to the equation (3.1), and $J \in C^{1}\left(H_{0}^{1}(\Omega), \mathbb{R}^{1}\right)$.

Besides, $J(0)=0$. And Sobolev embedding theorem yields that there is $c>0$ such that

$$
\begin{aligned}
J\left(u_{1}\right) & =\frac{1}{2} d_{1}\left\|u_{1}\right\|^{2}-\frac{1}{2} b_{1} \int_{\Omega} u_{1}^{2} d x+\frac{a_{11}}{2+\theta_{1}} \int_{\Omega} u_{1}^{2+\theta_{1}} d x \geqslant \frac{1}{2} d_{1}\left\|u_{1}\right\|^{2}-\frac{b_{1}}{2 \lambda_{1}}\left\|u_{1}\right\|^{2}-\frac{a_{11}}{2+\theta_{1}} \int_{\Omega}\left|u_{1}\right|^{2+\theta_{1}} d x \\
& \geqslant \frac{1}{2} d_{1}\left(1-\frac{b_{1}}{d_{1} \lambda_{1}}\right)\left\|u_{1}\right\|^{2}-\frac{c a_{11}}{2+\theta_{1}}\left\|u_{1}\right\|^{2+\theta_{1}} .
\end{aligned}
$$

Let $\rho>0$ small enough such that

$$
\left.J\right|_{\partial B_{\rho}(0)} \geqslant \alpha,
$$

where $\alpha=\frac{1}{2} d_{1}\left(1-\frac{b_{1}}{d_{1} \lambda_{1}}\right) \rho^{2}-\frac{c a_{11}}{2+\theta_{1}} \rho^{2+\theta_{1}}>0$. Denote by $\varphi_{1}(x)>0$ the eigenfunction of $\lambda_{1}$, satisfying $\left\|\varphi_{1}\right\|=1([11,17])$. Then

$$
J\left(-s \varphi_{1}\right)=\frac{1}{2} d_{1}\left\|-s \varphi_{1}\right\|^{2}-\frac{1}{2} b_{1} \int_{\Omega}\left(-s \varphi_{1}\right)^{2} d x+\frac{a_{11}}{2+\theta_{1}} \int_{\Omega}\left(-s \varphi_{1}\right)^{2+\theta_{1}} d x \rightarrow-\infty, \quad s \rightarrow+\infty,
$$

Thereby, there is a $s_{0}$ such that $s_{0}>\rho$ and $J\left(-s_{0} \varphi_{1}\right)<0$, where $\left\|-s_{0} \varphi_{1}\right\|=s_{0}>\rho$.

Let $\Gamma$ be the set of all paths connecting 0 and $-s_{0} \varphi_{1}$, i.e.,

$$
\Gamma=\left\{\psi \in C\left([0,1], H_{0}^{1}(\Omega)\right): \psi(0)=0, \psi(1)=-s_{0} \varphi_{1}\right\}
$$

Set

$$
c_{0}=\inf _{\psi \in \Gamma \in[0,1]} \max _{s \in[} J(\psi(s)) .
$$

then

$$
c_{0} \geqslant \frac{1}{2} d_{1}\left(1-\frac{b_{1}}{d_{1} \lambda_{1}}\right) \rho^{2}-\frac{c a_{11}}{2+\theta_{1}} \rho^{2+\theta_{1}}>0,
$$

Lemma 2.1 yields that there is a sequence $\left\{u_{1 n}\right\}_{n=1}^{\infty} \subset H_{0}^{1}(\Omega)$ such that

$$
J\left(u_{1 n}\right) \rightarrow c_{0}, \quad \text { and } \quad J^{\prime}\left(u_{1 n}\right) \rightarrow 0, \quad n \rightarrow \infty
$$

Below, similarly as those of [18], we will prove the sequence $\left\{u_{1 n}\right\}_{n=1}^{\infty} \subset H_{0}^{1}(\Omega)$ satisfying (3.10) must be bounded.

In fact, (3.10) yields

$$
\frac{1}{2} d_{1}\left\|u_{1 n}\right\|^{2}-\frac{1}{2} b_{1} \int_{\Omega} u_{1 n}^{2} d x+\frac{a_{11}}{2+\theta_{1}} \int_{\Omega} u_{1 n}^{2+\theta_{1}} d x=c_{0}+o(1)
$$

and

$$
d_{1}\left\|u_{1 n}\right\|^{2}-b_{1} \int_{\Omega} u_{1 n}^{2} d x+a_{11} \int_{\Omega} u_{1 n}^{2+\theta_{1}} d x=\left\langle J^{\prime}\left(u_{1 n}\right), u_{1 n}\right\rangle
$$

and for $\varepsilon>0$ small enough suc that there exits a $n$ big enough such that

$$
\left|\left\langle J^{\prime}\left(u_{1 n}\right), u_{1 n}\right\rangle\right| \leqslant \varepsilon\left\|u_{1 n}\right\|
$$

So we have

$$
d_{1}\left(\frac{1}{2}-\frac{1}{2+\theta_{1}}\right)\left(1-\frac{b_{1}}{d_{1} \lambda_{1}}\right)\left\|u_{1 n}\right\|^{2} \leqslant c_{0}+o(1)-\frac{\varepsilon}{2+\theta_{1}}\left\|u_{1 n}\right\|,
$$


which means the boundedness of $\left\{u_{1 n}\right\}_{n=1}^{\infty}$.

Now we shall prove that the bounded sequence $\left\{u_{1 n}\right\}_{n=1}^{\infty}$ must be compact sequentially. This is only a conventional proof. However, in view of the completeness of the proof, we are willing to give the proof:

In fact, (H1) means $\frac{1}{d_{1}}\left(b_{1} u_{1}(x)-a_{11} u_{1}(x)^{1+\theta_{1}}\right)$ satisfies the Caratheodory condition:

$$
\left|\frac{1}{d_{1}}\left(b_{1} u_{1}(x)-a_{11} u_{1}(x)^{1+\theta_{1}}\right)\right| \leqslant c_{1}+c_{2}\left|u_{1}\right|^{2}, \quad \forall\left(x, u_{1}\right) \in \Omega \times \mathbb{R}
$$

where $c_{1}, c_{2}$ are positive numbers big enough. Due to $\Omega \subset \mathbb{R}^{3}$, then the critical Sobolev exponent is 6 , and hence the operator $J^{\prime}: H_{0}^{1}(\Omega) \rightarrow\left(H_{0}^{1}(\Omega)\right)^{*}$ is compact, where the functional

$$
\tilde{J}=\int_{\Omega}\left(\frac{1}{2} b_{1} u_{1}^{2}-\frac{a_{11}}{2+\theta_{1}} u_{1}^{2+\theta_{1}}\right) d x .
$$

Moreover,

$$
\left\langle\tilde{J}^{\prime}\left(u_{1}\right), \varphi\right\rangle=\int_{\Omega}\left(b_{1} u_{1}(x) \varphi-a_{11} u_{1}(x)^{1+\theta_{1}} \varphi\right) d x, \quad \forall \varphi \in H_{0}^{1}(\Omega) .
$$

and then the bounded sequence $\left\{u_{1 n}\right\}_{n=1}^{\infty}$ possesses a subsequence, say, $\left\{u_{1 n}\right\}_{n=1}^{\infty}$, satisfying $J^{\prime}\left(u_{1 n}\right) \rightarrow J^{\prime}\left(u_{1 *}\right)$ in $\left(H_{0}^{1}(\Omega)\right)^{*}, n \rightarrow \infty$, where $u_{1 *} \in H_{0}^{1}(\Omega)$. For any $\varphi \in H_{0}^{1}(\Omega)$,

$$
\left\langle J^{\prime}\left(u_{1 n}\right)-J^{\prime}\left(u_{1 m}\right), \varphi\right\rangle=d_{1} \int_{\Omega}\left(\nabla u_{1 n}-\nabla u_{1 m}\right) \cdot \nabla \varphi d x-\left\langle\tilde{J}^{\prime}\left(u_{1 n}\right)-\tilde{J}^{\prime}\left(u_{1 m}\right), \varphi\right\rangle,
$$

which together with $\left\{u_{1 n}\right\}_{n=1}^{\infty} \subset H_{0}^{1}(\Omega),(3.10)$ and the arbitrariness of $\varphi$ implies

$$
\begin{gathered}
\left\|u_{1 n}-u_{1 m}\right\|^{2} \leqslant\left(\left\|J^{\prime}\left(u_{1 n}\right)\right\|+\left\|J^{\prime}\left(u_{1 m} \|\right)\right\| u_{1 n}-u_{1 m}\|+\| \tilde{J}^{\prime}\left(u_{1 n}\right)-\tilde{J}^{\prime}\left(u_{1 m}\right)\|\| u_{1 n}-u_{1 m} \|\right. \\
\leqslant\left(\left\|J^{\prime}\left(u_{1 n}\right)\right\|+\| J^{\prime}\left(u_{1 m}\|+\| \tilde{J}^{\prime}\left(u_{1 n}\right)-\tilde{J}^{\prime}\left(u_{1 m}\right) \|\right)\left(\left\|u_{1 n}\right\|+\left\|u_{1 m}\right\|\right) \rightarrow 0, \quad n \rightarrow \infty, m \rightarrow \infty,\right.
\end{gathered}
$$

This shows that $\left\{u_{1 n}\right\}_{n=1}^{\infty}$ is compact sequentially. And then there exists a subsequence of $\left\{u_{1 n}\right\}_{n=1}^{\infty}$ convergent to a point in $H_{0}^{1}(\Omega)$, say, $u_{1 *} \in H_{0}^{1}(\Omega)$, Due to $J\left(u_{1 *}\right)=c_{0} \geqslant \frac{1}{2} d_{1}\left(1-\frac{b_{1}}{d_{1} \lambda_{1}}\right) \rho^{2}-\frac{c a_{11}}{2+\theta_{1}} \rho^{2+\theta_{1}}>0$, we see $u_{1 *} \neq 0$, which shows that $\left(u_{1 *}, 0\right) \neq(0,0)$. Similarly, we can similarly prove there is at least another stationary solution $\left(0, u_{2 *}\right) \neq(0,0)$ for the system $(2.1)$.

Remark 3. Due to the mathematical difficulty brought about by Dirichlet zero-boundary value (see (I3) for details), we have to employ variational methods to overcome it, in which the variational technique developed in [18] ploys an important role. In sum, the difficulty is much bigger than that of Neumann zero-boundary value in many previous literature.

Remark 4. In Theorem 3.1, the existence of multiple stationary solutions illuminates that the zero solution $(0,0)$ can not be global stable under the conditions of Theorem 3.1. And so only the local stability may be considered below. But it is worth mentioning that the conditions of Theorem 3.1 directly warrant the local stability of the zero solution (see Theorem 3.2), which directly shows that the sufficient condition on the existence of multiple stationary solutions of RDGACM in Theorem 3.1 is just perfect.

Theorem 3.2. Under the assumptions of Theorem 3.1, the zero solution $(0,0)$ of the RDGACM (2.1) is locally asymptotically stable.

Proof. Firstly, the condition $b_{i}<\lambda_{1} d_{i}$ yields,

$$
B<\lambda_{1} D,
$$

where

$$
D=\left(\begin{array}{ll}
d_{1} & 0 \\
0 & d_{2}
\end{array}\right), B=\left(\begin{array}{cc}
b_{1} & 0 \\
0 & b_{2}
\end{array}\right) \text {. }
$$


Next, consider the following linear system:

Consider the Lyapunov function:

$$
\left\{\begin{array}{l}
\frac{\partial u_{1}}{\partial t}=d_{1} \Delta u_{1}+b_{1} u_{1}, \quad t \geqslant 0, x \in \Omega \\
\frac{\partial u_{2}}{\partial t}=d_{2} \Delta u_{2}+b_{2} u_{2}, \quad t \geqslant 0, x \in \Omega \\
u_{1}(t, x)=u_{2}(t, x)=0, \quad t \geqslant 0, x \in \partial \Omega \\
u_{1}(0, x)=\xi_{1}(x), \quad u_{2}(0, x)=\xi_{2}(x)
\end{array}\right.
$$

The condition (3.14) yields

$$
V=\int_{\Omega}\left(u_{1}^{2}+u_{2}^{2}\right) d x
$$

$$
\begin{aligned}
\left.\frac{d V}{d t}\right|_{(3.16)} & =\int_{\Omega}\left(2 d_{1} u_{1} \Delta u_{1}+2 b_{1} u_{1}^{2}+2 d_{2} u_{2} \Delta u_{2}+2 b_{2} u_{2}^{2}\right) d x \\
& \leqslant \int_{\Omega} u^{T}\left(-2 \lambda_{1} D+2 B\right) u d x \leqslant 0,
\end{aligned}
$$

where $u=\left(u_{1}, u_{2}\right)^{T}$. Then (3.17) yields that the zero solution $(0,0)$ of the linear system (3.16) is asymptotically stable ([19]). And hence, the zero solution $(0,0)$ of the nonlinear system $(2.1)$ is locally asymptotically stable.

Remark 5. The main purpose is to investigate the ecosystem on harmful species, and so only the stability of the zero solution is considered. The conditions of Theorem 3.1 perfectly warrant the local stability of the zero solution. And such conditions give us a useful hint on how to eliminate pests to some extent. Under the conditions of Theorem 3.2, we know, if the initial value is in the domain of attraction of the zero solution, both of two population densities will eventually tend to zero.

\section{Global Stability with boundedness assumption on population densities}

Theorem 3.2 illuminates that under the conditions of Theorem 3.2 or Theorem 3.1, a suitable initial value will make two population densities tend to zero eventually. In this section, we want to find out such suitable conditions that two population densities eventually tend to zero, no matter what the initial value is. So in this section, we do not need the condition (H1). Another suitable assumption may proposed on population densities $u_{1}, u_{2}$ :

$$
0 \leqslant u_{1} \leqslant M_{1}, \quad 0 \leqslant u_{2} \leqslant M_{2},
$$

where $M_{i}$ is a given positive number for a given $i \in\{1,2\}$.

Remark 6. This assumption is reasonable due to the limited resources (see, e.g. [20]).

Next, the system (2.1) can be rewrite as follows,

$$
\left\{\begin{array}{l}
\frac{\partial u_{1}}{\partial t}=d_{1} \Delta u_{1}+b_{1} u_{1}-a_{11} f_{1}\left(u_{1}\right)+0 \cdot f_{2}\left(u_{2}\right), \quad t \geqslant 0, x \in \Omega \\
\frac{\partial u_{2}}{\partial t}=d_{2} \Delta u_{2}+b_{2} u_{2}+0 \cdot f_{1}\left(u_{1}\right)-a_{22} f_{2}\left(u_{2}\right), \quad t \geqslant 0, x \in \Omega \\
u_{1}(t, x)=u_{2}(t, x)=0, \quad t \geqslant 0, x \in \partial \Omega, \\
u_{1}(0, x)=\xi_{1}(x), \quad u_{2}(0, x)=\xi_{2}(x)
\end{array}\right.
$$

where

$$
f_{1}\left(u_{1}\right)=u_{1}^{1+\theta_{1}}+\frac{a_{12}}{a_{11}} u_{1} u_{2}
$$




$$
f_{2}\left(u_{2}\right)=u_{2}^{1+\theta_{2}}+\frac{a_{21}}{a_{22}} u_{1} u_{2} .
$$

And the system (4.2) can be rewritten as follows,

$$
\left\{\begin{aligned}
\frac{\partial u}{\partial t} & =D \Delta u+B u-A f(u), \quad t \geqslant 0, x \in \Omega, \\
u(t, x) & =0, \quad t \geqslant 0, x \in \partial \Omega \\
u(0, x) & =\xi(x), \quad x \in \Omega
\end{aligned}\right.
$$

where the matrices $D$ and $B$ is defined in (3.15), $u=\left(u_{1}, u_{2}\right)^{T}, f(u)=\left(f_{1}\left(u_{1}\right), f_{2}\left(u_{2}\right)\right)^{T}, \xi=\left(\xi_{1}, \xi_{2}\right)^{T}$, and

$$
A=\left(\begin{array}{cc}
a_{11} & 0 \\
0 & a_{22}
\end{array}\right) \text {. }
$$

Moreover, since the larva usually does not have the competitive ability, and the larva matures to the adult needs a period of time, which is usually closely related to the climate, temperature, humidity and other random factors, so the delayed feedback stochastic model may be considered ([20]). Denote by $(\Upsilon, \mathcal{F}, \mathbb{P})$ the complete probability space with a natural filtration $\left\{\mathcal{F}_{t}\right\}_{t \geq 0}$ (see, e.g. [22]). Let $S=\left\{1,2, \cdots, n_{0}\right\}$ and the random form process $\{r(t):[0,+\infty) \rightarrow S\}$ be a homogeneous, finite-state Markovian process with right continuous trajectories with generator $\Pi=\left(\gamma_{i j}\right)_{n_{0} \times n_{0}}$ and transition probability from mode $i$ at time $t$ to mode $j$ at time $t+\delta, i, j \in S$,

$$
\mathbb{P}(r(t+\delta)=j \mid r(t)=i)=\left\{\begin{array}{l}
\gamma_{i j} \delta+o(\delta), \quad j \neq i \\
1+\gamma_{i j} \delta+o(\delta), \quad j=i
\end{array}\right.
$$

where $\gamma_{i j} \geqslant 0$ is transition probability rate from $i$ to $j(j \neq i)$ and $\gamma_{i i}=-\sum_{j=1, j \neq i}^{n_{0}} \gamma_{i j}, \delta>0$ and $\lim _{\delta \rightarrow 0} o(\delta) / \delta=0$.

Consider the following delayed feedback system :

$$
\left\{\begin{aligned}
\frac{\partial u(t, x)}{\partial t} & =D \Delta u(t, x)+B u(t, x)-A f(u(t, x))+K(r(t))(u(t, x)-u(t-\tau(r(t), t), x)), \quad t \geqslant 0, x \in \Omega, \\
u(t, x) & =0, \quad t \geqslant 0, x \in \partial \Omega \\
u(s, x) & =\xi(s, x), \quad x \in \Omega, s \in[0, \tau]
\end{aligned}\right.
$$

where $\xi(s, x)=\left(\xi_{1}(s, x), \xi_{2}(s, x)\right)^{T} \in \mathbb{R}^{2}$ is bounded and continuous.

$$
K_{r}=K(r(t))=\left(\begin{array}{cc}
k_{1}(r(t)) & 0 \\
0 & k_{2}(r(t))
\end{array}\right),
$$

$k_{1}(r(t))$ and $k_{2}(r(t))$ are feedback benefit coefficients at mode $r(t)=r \in S$. Denote $k_{1}(r(t))=k_{1 r}, k_{2}(r(t))=k_{2 r}$ for simple.

Theorem 4.1. If

$$
\lambda_{1} D>B+A F
$$

then the null solution is the unique stationary solution of the delayed RDGACM (4.7) under the restrictive condition (4.1). Moreover, if

$$
1-\tau_{*}-\sum_{j \in S} \gamma_{r j} \tau>0
$$


and there is a positive number $\beta$, a sequences of positive definite diagonal matrices $P_{r}(r \in S), Q_{i}>0(i \in\{1,2\})$ and $W>0$ such that

$$
\left(\begin{array}{cccc}
-\Theta_{r} & \frac{1}{2} F Q_{1}-P_{r} A & -P_{r} K_{r} & 0 \\
* & -Q_{1} & 0 & 0 \\
* & * & -\left(1-\tau_{*}-\sum_{j \in S} \gamma_{r j} \tau\right) e^{-\beta \tau} W & \frac{1}{2} F Q_{2} \\
* & * & * & -Q_{2}
\end{array}\right)<0
$$

then the null solution of the delayed RDGACM (4.7) is globally exponentially stable with convergence rate $\frac{\beta}{2}$, where $\tau_{r}(t) \in[0, \tau]$ with $\dot{\tau}_{r}(t) \leqslant \tau_{*}$

$$
\Theta_{r}=2 \lambda_{1} P_{r} D-2 P_{r} B-\sum_{j \in S} \gamma_{r j} P_{j}-2 K_{r} P_{r}-\beta P_{r}-W,
$$

and

$$
F=\left(\begin{array}{cc}
F_{1} & 0 \\
0 & F_{2}
\end{array}\right)
$$

with

$$
\begin{aligned}
& F_{1}=\left(1+\theta_{1}\right) M_{1}^{\theta_{1}}+\frac{a_{12}}{a_{11}} M_{2} \\
& F_{2}=\left(1+\theta_{2}\right) M_{2}^{\theta_{2}}+\frac{a_{21}}{a_{22}} M_{1} .
\end{aligned}
$$

Proof. Firstly, it follows from (4.1) and (4.3)-(4.4) that $f_{i}(0)=0, i=1,2$, and

$$
0 \leqslant \frac{f_{1}(r)-f_{1}(s)}{r-s} \leqslant\left(1+\theta_{1}\right) M_{1}^{\theta_{1}}+\frac{a_{12}}{a_{11}} M_{2}
$$

and

$$
0 \leqslant \frac{f_{2}(r)-f_{2}(s)}{r-s} \leqslant\left(1+\theta_{2}\right) M_{2}^{\theta_{2}}+\frac{a_{21}}{a_{22}} M_{1} .
$$

Next, one can see that under the restrictive condition $(4.1)$ on the state variable $u$, the null solution $(0,0)$ is the unique stationary solution of the system (4.7)

Indeed, let $u \equiv u(x)$ be a stationary solution, satisfying (4.1), then it is obvious that

$$
0=D \Delta u+B u-A f(u)
$$

which together with the definition of $f$, Poincare inequality and boundary value condition implies

$$
\int_{\Omega}|u|^{T}(B+A F)|u| d x \geqslant \int_{\Omega}\left(|u|^{T} B|u|+|u|^{T} A|f(u)|\right) d x \geqslant \lambda_{1} \int_{\Omega}|u|^{T} D|u| d x .
$$

Combining (4.8) and (4.16) results in $u=0$, and hence, the null solution solution must be the unique stationary solution of the ecosystem (4.7) under the restrictive condition (4.1) on the state variable $u$.

Let $P_{r}(r \in S)$ and $W$ be positive definite matrices such that

$$
V(t, r, u)=\int_{\Omega} e^{\beta t} u^{T}(t, x) P_{r} u(t, x) d x+\int_{t-\tau(r(t), t)}^{t} \int_{\Omega} e^{\beta s} u^{T}(s, x) W u(s, x) d x d s .
$$

On the other hand, it follows from (4.13) and (4.14) that

$$
\left(u^{T}, f(u)^{T}\right)\left(\begin{array}{cc}
0 & \frac{1}{2} F Q_{1} \\
* & -Q_{1}
\end{array}\right)\left(\begin{array}{c}
u \\
f(u)
\end{array}\right) \geqslant 0,
$$


similarly,

$$
\left(u^{T}\left(t-\tau_{r}(t), x\right), f^{T}\left(u\left(t-\tau_{r}(t), x\right)\right)\right)\left(\begin{array}{cc}
0 & \frac{1}{2} F Q_{2} \\
* & -Q_{2}
\end{array}\right)\left(\begin{array}{c}
u\left(t-\tau_{r}(t), x\right) \\
f\left(u\left(t-\tau_{r}(t), x\right)\right)
\end{array}\right) \geqslant 0,
$$

Let $\mathcal{L}$ be the weak infinitesimal operator (see, e.g. [15]) such that

$$
\begin{aligned}
& \mathcal{L} V \leqslant-e^{\beta t}\left[\int_{\Omega} u^{T}\left(2 \lambda_{1} P_{r} D-2 P_{r} B-\sum_{j \in S} \gamma_{r j} P_{j}-2 K_{r} P_{r}-\beta P_{r}-W\right) u d x+2 \int_{\Omega} u^{T} P_{r} A f(u) d x\right. \\
& \left.+2 \int_{\Omega} u^{T} P_{r} K_{r} u\left(t-\tau_{r}(t), x\right) d x+\left(1-\tau_{*}-\sum_{j \in S} \gamma_{r j} \tau\right) e^{-\beta \tau} \int_{\Omega} u^{T}\left(t-\tau_{r}(t), x\right) W u\left(t-\tau_{r}(t), x\right) d x\right] \\
& \leqslant e^{\beta t}\left[-\int_{\Omega} u^{T}\left(2 \lambda_{1} P_{r} D-2 P_{r} B-\sum_{j \in S} \gamma_{r j} P_{j}-2 K_{r} P_{r}-\beta P_{r}-W\right) u d x-2 \int_{\Omega} u^{T} P_{r} A f(u) d x\right. \\
& -2 \int_{\Omega} u^{T} P_{r} K_{r} u\left(t-\tau_{r}(t), x\right) d x-\left(1-\tau_{*}-\sum_{j \in S} \gamma_{r j} \tau\right) e^{-\beta \tau} \int_{\Omega} u^{T}\left(t-\tau_{r}(t), x\right) W u\left(t-\tau_{r}(t), x\right) d x \\
& \left.+\left(\begin{array}{c}
u \\
f(u)
\end{array}\right)^{T}\left(\begin{array}{cc}
0 & \frac{1}{2} F Q_{1} \\
* & -Q_{1}
\end{array}\right)\left(\begin{array}{c}
u \\
f(u)
\end{array}\right)+\left(\begin{array}{c}
u\left(t-\tau_{r}(t), x\right) \\
f\left(u\left(t-\tau_{r}(t), x\right)\right)
\end{array}\right)^{T}\left(\begin{array}{cc}
0 & \frac{1}{2} F Q_{2} \\
* & -Q_{2}
\end{array}\right)\left(\begin{array}{c}
u\left(t-\tau_{r}(t), x\right) \\
f\left(u\left(t-\tau_{r}(t), x\right)\right)
\end{array}\right)\right] \\
& =e^{\beta t}\left(\begin{array}{c}
u \\
f(u) \\
u\left(t-\tau_{r}(t), x\right) \\
f\left(u\left(t-\tau_{r}(t), x\right)\right)
\end{array}\right)^{T}\left(\begin{array}{cccc}
-\Theta_{r} & \frac{1}{2} F Q_{1}-P_{r} A & -P_{r} K_{r} & 0 \\
* & -Q_{1} & 0 & 0 \\
* & * & -\left(1-\tau_{*}-\sum_{j \in S} \gamma_{r j} \tau\right) e^{-\beta \tau} W & \frac{1}{2} F Q_{2} \\
* & * & * & -Q_{2}
\end{array}\right)\left(\begin{array}{c}
u \\
f(u) \\
u\left(t-\tau_{r}(t), x\right) \\
f\left(u\left(t-\tau_{r}(t), x\right)\right) .
\end{array}\right) \leqslant 0 .
\end{aligned}
$$

Repeating the steps similar as those of [15, (3.16)-(3.17)] results in the conclusion of Theorem 4.1. For the integrity of the proof, we are willing to rewrite it simply. For any $\left(\xi_{1}(s, x), \xi_{2}(s, x)\right)^{T} \in L_{\mathcal{F}_{0}}^{2}\left([-\tau, 0] \times \Omega ; \mathbb{R}^{2}\right)$ and any mode $r \in S$, we see it from (4.17) that for the solution $u_{1}\left(t, x ; \xi_{1}, r_{0}\right), u_{2}\left(t, x ; \xi_{2}, r_{0}\right)$ of the system $(4.7)$ with initial value $\left(\xi_{1}, \xi_{2}\right)^{T}$, there are two positive scalars $c_{i}>0(i=1,2)$ independent of any mode $r \in S$ such that

$$
\begin{aligned}
& c_{1} e^{\beta t}\left(\mathbb{E}\left\|u_{1}\left(t, \xi_{1}\right)\right\|_{L^{2}(\Omega)}^{2}+\mathbb{E}\left\|u_{2}\left(t, \xi_{2}\right)\right\|_{L^{2}(\Omega)}^{2}\right) \\
\leqslant & \mathbb{E} V\left(t, u_{1}(t, x), u_{2}(t, x), r(t)\right) \leqslant \mathbb{E} V\left(t_{0}, u_{1}\left(t_{0}, x\right), u_{2}\left(t_{0}, x\right), r\left(t_{0}\right)\right) \\
\leqslant & c_{2}\left(\sup _{-\tau \leqslant s \leqslant 0} \mathbb{E}\left(\left\|\xi_{1}(s)\right\|_{L^{2}(\Omega)}^{2}\right)+\sup _{-\tau \leqslant s \leqslant 0} \mathbb{E}\left(\left\|\xi_{2}(s)\right\|_{L^{2}(\Omega)}^{2}\right),\right.
\end{aligned}
$$

or

$$
\mathbb{E}\left\|u_{1}\left(t, \xi_{1}\right)\right\|_{L^{2}(\Omega)}^{2}+\mathbb{E}\left\|u_{2}\left(t, \xi_{2}\right)\right\|_{L^{2}(\Omega)}^{2} \leqslant \frac{c_{2}}{c_{1}} e^{\beta t}\left(\sup _{-\tau \leqslant s \leqslant 0} \mathbb{E}\left(\left\|\xi_{1}(s)\right\|_{L^{2}(\Omega)}^{2}\right)+\sup _{-\tau \leqslant s \leqslant 0} \mathbb{E}\left(\left\|\xi_{2}(s)\right\|_{L^{2}(\Omega)}^{2}\right)\right),
$$

which has proved that the null solution of the ecosystem (4.7) is globally exponentially stable with convergence rate $\frac{\beta}{2}$. Here, we denote $r_{0}=r(0)$ for simple.

Remark 7. The LMI-based stability criterion and free weight coefficient matrix make Theorem 4.1 reduce the conservatism of the algorithm.

Remark 8. No matter what the initial value is, both of two population densities must eventually tend to zero under the conditions of Theorem 4.1, which gives us effective tips on how to eliminate pests.

Remark 9. In the neural networks system [27, (1)], the self-feedback term $A$ is a positive definite matrix such that $-A$ is a negative definite matrix. But in our ecosystem (4.7) or (2.1), there is not such a similar negative definite matrix. Note the LMI condition (4.10) means a big negative definite matrix is necessary. And so the LMI condition (4.10) is harsh to some extent. But in Theorem 
3.2, the sufficient conditions of local stability is not harsh at all. This implies that if we want to exterminate destructive insects inevitably no matter what the initial value is, we need human intervention to some extent. Impulse control technique should be considered. Besides, it is also an unreasonable assumption that time delay must be differentiable. So we shall abandon the abovementioned two unreasonable assumptions about negative definite matrix and differentiable delay time functions in a new theorem as follows.

Consider the following impulsive RDGACM:

$$
\left\{\begin{aligned}
\frac{\partial u(t, x)}{\partial t} & =D \Delta u(t, x)+B u(t, x)-A f(u(t, x))+K(r(t))(u(t, x)-u(t-\tau(r(t), t), x)), \quad t \geqslant 0, t \neq t_{k}, x \in \Omega, \\
u\left(t_{k}^{+}, x\right) & =u\left(t_{k}, x\right)=\mathcal{M}_{k} u\left(t_{k}^{-}, x\right), \quad k=1,2, \cdots, x \in \Omega \\
u(t, x) & =0, \quad t \geqslant 0, x \in \partial \Omega, \\
u(s, x) & =\xi(s, x), \quad x \in \Omega, s \in[0, \tau]
\end{aligned}\right.
$$

where each $\mathcal{M}_{k}$ is a positive definite diagonal matrix with $\lambda_{\max } \mathcal{M}_{k}<1$ for $k \in \mathbb{Z}^{+}$.

Theorem 4.2. Suppose the boundedness condition (4.1) holds. If there exits a positive definite diagonal matrix $\Lambda_{1}>0$ such that

$$
B+\frac{1}{2} \Lambda_{1} A^{2}+\frac{1}{2} \Lambda_{1}^{-1} F^{2}<\lambda_{1} D
$$

then the null solution is the unique stationary solution of RDGACM (4.20). Moreover, assume that $\sup _{k \in \mathbb{Z}^{+}}\left(t_{k}-t_{k-1}\right)<+\infty$. Besides, there exist positive scalars $\sigma, \lambda>0, \gamma \geqslant 1$ and positive definite diagonal matrices $P_{r}(r \in S)$ such that

$$
\max _{r \in S}\left(\frac{\mu_{r}}{\lambda_{\min } P_{r}}\right)+\gamma e^{\lambda \tau} \max _{r \in S}\left(\frac{\lambda_{\max } \Lambda_{3}^{-1}}{\lambda_{\min } P_{r}}\right) \leqslant \sigma-\lambda
$$

(H2) each $\mathcal{M}_{k}$ is a positive definite diagonal matrix with $\lambda_{\max } \mathcal{M}_{k}<1$ for $k \in \mathbb{Z}^{+}$.

(H3) $\gamma \geqslant \frac{1}{\lambda_{\max } \mathcal{M}_{k}^{2}}$ and $\lambda_{\max } \mathcal{M}_{k}^{2}<e^{-(\sigma+\lambda)\left(t_{k}-t_{k-1}\right)}, \forall k \in \mathbb{Z}^{+}$

then the null solution of RDGACM (4.20) is globally exponentially stable with convergence rate $\frac{\lambda}{2}$.

Proof. Firstly, one can prove that under the restrictive condition (4.1) on the state variable $u$, the null solution $(0,0)$ is the unique stationary solution of the RDGACM (4.20).

Indeed, assume $u \equiv u(x)$ is a stationary solution, satisfying (4.1), then

$$
\begin{gathered}
0=D \Delta u+B u-A f(u) . \\
\int_{\Omega}\left(u^{T} B u-u^{T} A f(u)\right) d x=-D \int_{\Omega} u^{T} \Delta u d x=\int_{\Omega}|\nabla u|^{T} D|\nabla u| d x \geqslant \lambda_{1} \int_{\Omega} u^{T} D u d x .
\end{gathered}
$$

On the other hand, since $A$ is a diagonal matrix,

$$
2\left[-u^{T} A f(u)\right]=(-A u)^{T} f(u)+f^{T}(u)(-A u) \leqslant(-A u)^{T} \Lambda_{1}(-A u)+f^{T}(u) \Lambda_{1}^{-1} f(u) \leqslant u^{T} \Lambda_{1} A^{2} u+u^{T} \Lambda_{1}^{-1} F^{2} u,
$$

which implies

$$
\int_{\Omega} u^{T}\left(B+\frac{1}{2} \Lambda_{1} A^{2}+\frac{1}{2} \Lambda_{1}^{-1} F^{2}\right) u d x \geqslant \lambda_{1} \int_{\Omega} u^{T} D u d x
$$

(4.24) together with the condition (4.21) means that the null solution is the unique stationary solution of RDGACM (4.20).

Below, we only need to prove the global exponential stability of $(0,0)^{T}$ under the impulse control. Consider the Lyapunov function as follows, 


$$
\begin{gathered}
V(t)=\int_{\Omega} u^{T}(t, x) P_{r} u(t, x) d x . \\
c_{1}\|u\|_{L^{2}(\Omega)}^{2} \leqslant V(t)=\int_{\Omega} u^{T}(t, x) P_{r} u(t, x) d x \leqslant c_{2}\|u\|_{L^{2}(\Omega)}^{2} \\
c_{1}=\min _{r \in S} \lambda_{\min } P_{r}>0, \quad c_{2}=\max _{r \in S} \lambda_{\max } P_{r}>0,
\end{gathered}
$$

Let $\mathcal{L}$ be the weak infinitesimal operator such that

$$
\mathcal{L} V \leqslant \max _{r \in S}\left(\frac{\mu_{r}}{\lambda_{\min } P_{r}}\right) \int_{\Omega} u^{T} P_{r} u d x+\max _{r \in S}\left(\frac{\lambda_{\max } \Lambda_{3}^{-1}}{\lambda_{\min } P_{r}}\right) \int_{\Omega} u^{T}\left(t-\tau_{r}(t), x\right) P_{r} u\left(t-\tau_{r}(t), x\right) d x
$$

where

$$
\mu_{r}=\lambda_{\max }\left(-2 \lambda_{1} P_{r} D+2 P_{r} B+\sum_{j \in S} \gamma_{r j} P_{j}+2 K_{r} P_{r}+\Lambda_{2} P_{r}^{2} A^{2}+\Lambda_{2}^{-1} F^{2}+\Lambda_{3} P_{r}^{2} K_{r}^{2}\right)
$$

Moreover,

$$
\mathbb{E} V(t+\varepsilon)-\mathbb{E} V(t)=\int_{t}^{t+\varepsilon} \mathbb{E} \mathcal{L} V(s) d s
$$

Let $\varepsilon \rightarrow 0$, then (4.26) leads to

$$
D^{+} \mathbb{E} V \leqslant \max _{r \in S}\left(\frac{\mu_{r}}{\lambda_{\min } P_{r}}\right) \mathbb{E} \int_{\Omega} u^{T} P_{r} u d x+\max _{r \in S}\left(\frac{\lambda_{\max } \Lambda_{3}^{-1}}{\lambda_{\min } P_{r}}\right) \mathbb{E} \int_{\Omega} u^{T}\left(t-\tau_{r}(t), x\right) P_{r} u\left(t-\tau_{r}(t), x\right) d x
$$

Next, we claim that

$$
\mathbb{E} V(t) \leqslant M\|\xi\|_{\tau}^{2} e^{-\lambda\left(t-t_{0}\right)}, \quad \forall t \in\left[t_{k-1}, t_{k}\right), k \in \mathbb{Z}^{+}
$$

where

$$
\|\xi\|_{\tau}^{2}=\sup _{s \in[-\tau, 0]} \int_{\Omega}|\xi 1(s, x)|^{2} d x+\sup _{s \in[-\tau, 0]} \int_{\Omega}\left|\xi_{2}(s, x)\right|^{2} d x .
$$

Indeed, employing mathematical induction will lead to (2).

It follows from (H3) that there exists a positive constant $M>0$ satisfying

$$
0<c_{2} e^{(\sigma+\lambda)\left(t_{1}-t_{0}\right)} \leqslant M \leqslant c_{2} \gamma e^{\lambda \tau-(\sigma+\lambda)\left(t_{1}-t_{0}\right)} e^{(\sigma+\lambda)\left(t_{1}-t_{0}\right)}
$$

And then

$$
0<c_{2}\|\xi\|_{\tau}^{2}<c_{2}\|\xi\|_{\tau}^{2} e^{\sigma\left(t_{1}-t_{0}\right)} \leqslant M\|\xi\|_{\tau}^{2} e^{-\lambda\left(t_{1}-t_{0}\right)}
$$

At first, we need to prove

$$
\mathbb{E} V(t) \leqslant M\|\xi\|_{\tau}^{2} e^{-\lambda\left(t-t_{0}\right)}, \quad \forall t \in\left[t_{0}, t_{1}\right)
$$

which implies that we only need to show

$$
\mathbb{E} V(t) \leqslant M\|\xi\|_{\tau}^{2} e^{-\lambda\left(t_{1}-t_{0}\right)}, \quad \forall t \in\left[t_{0}, t_{1}\right)
$$

In fact, it is obvious from (4.25) that

$$
\mathbb{E} V\left(t_{0}\right) \leqslant c_{2}\left\|u\left(t_{0}\right)\right\|_{L^{2}(\Omega)}^{2} \leqslant c_{2}\|\xi\|_{\tau}^{2}<c_{2} e^{\sigma\left(t_{1}-t_{0}\right)}\|\xi\|_{\tau}^{2} \leqslant M\|\xi\|_{\tau}^{2} e^{-\lambda\left(t_{1}-t_{0}\right)} .
$$

Thus, if (6) does not hold, there must exist some $t \in\left(t_{0}, t_{1}\right)$ such that

$$
\mathbb{E} V(t)>M\|\xi\|_{\tau}^{2} e^{-\lambda\left(t_{1}-t_{0}\right)},
$$


which implies that there is $t^{*} \in\left(t_{0}, t_{1}\right)$, satisfying

$$
\mathbb{E} V\left(t^{*}\right)=M\|\xi\|_{\tau}^{2} e^{-\lambda\left(t_{1}-t_{0}\right)}, \quad \text { and } \mathbb{E} V(t) \leqslant M\|\xi\|_{\tau}^{2} e^{-\lambda\left(t_{1}-t_{0}\right)}=\mathbb{E} V\left(t^{*}\right), \forall t \in\left[t_{0}, t^{*}\right),
$$

which together with (4.25) and (*) means that $\mathbb{E} V\left(t_{0}\right) \leqslant c_{2}\|\xi\|_{\tau}^{2}<M\|\xi\|_{\tau}^{2} e^{-\lambda\left(t_{1}-t_{0}\right)}$, and hence, there is $t^{* *} \in\left[t_{0}, t^{*}\right)$ such that $\mathbb{E} V\left(t^{* *}\right)=c_{2}\|\xi\|_{\tau}^{2}$ and

$$
\mathbb{E} V\left(t^{* *}\right) \leqslant \mathbb{E} V(t) \leqslant \mathbb{E} V\left(t^{*}\right), \quad \forall t \in\left[t^{* *}, t^{*}\right]
$$

On the other hand, (4.25) yield

$$
\begin{aligned}
\mathbb{E} V\left(t^{*}\right) & \leqslant \mathbb{E} V\left(t^{* *}\right) e^{(\sigma-\lambda)\left(t^{*}-t^{* *}\right)} \\
& <\mathbb{E} V\left(t^{* *}\right) e^{\sigma\left(t_{1}-t_{0}\right)} \quad \leqslant M\|\xi\|_{\tau}^{2} e^{-\lambda\left(t_{1}-t_{0}\right)}=\mathbb{E} V\left(t^{*}\right)
\end{aligned}
$$

This contradiction implies that (6) holds, and then (5) holds.

Next, we assume that (2) holds for $k=1,2, \cdots, m$, or

$$
\mathbb{E} V(t) \leqslant M\|\xi\|_{\tau}^{2} e^{-\lambda\left(t-t_{0}\right)}, \quad \forall t \in\left[t_{k-1}, t_{k}\right), k=1,2, \cdots, m
$$

Below, we shall conclude

$$
\mathbb{E} V(t) \leqslant M\|\xi\|_{\tau}^{2} e^{-\lambda\left(t-t_{0}\right)}, \quad \forall t \in\left[t_{m}, t_{m+1}\right)
$$

It is obvious that

$$
\mathbb{E} V\left(t_{m}\right)<M\|\xi\|_{\tau}^{2} e^{-\lambda\left(t_{m}-t_{0}\right)} .
$$

Indeed, since $\mathcal{M}_{m}$ is a diagonal matrix,

$$
\begin{aligned}
\mathbb{E} V\left(t_{m}\right) & =\mathbb{E} \int_{\Omega} u^{T}\left(t_{m}, x\right) P_{r} u\left(t_{m}, x\right) d x \\
& =\mathbb{E} \int_{\Omega} u^{T}\left(t_{m}^{+}, x\right) P_{r} u\left(t_{m}^{+}, x\right) d x \\
& <M\|\xi\|_{\tau}^{2} e^{-\lambda\left(t_{m}-t_{0}\right)}
\end{aligned}
$$

Suppose (11) is not true. Define $t_{b}=\inf \left\{t \in\left[t_{m}, t_{m+1}\right): \mathbb{E} V(t)>M\|\xi\|_{\tau}^{2} e^{-\lambda\left(t-t_{0}\right)}\right\}$. Then the continuity of $\mathbb{E} V(t)$ on $\left[t_{m}, t_{m+1}\right)$ derives

$$
\mathbb{E} V\left(t_{b}\right)=M\|\xi\|_{\tau}^{2} e^{-\lambda\left(t_{b}-t_{0}\right)} \quad \text { and } \quad \mathbb{E} V(t) \leqslant M\|\xi\|_{\tau}^{2} e^{-\lambda\left(t-t_{0}\right)}, \forall t \in\left[t_{m}, t_{b}\right]
$$

And $(* * *)$ yields $t_{m} \neq t_{b}$, and hence $t_{m}<t_{b}<t_{m+1}$.

On the other hand,

$$
\begin{aligned}
\mathbb{E} V\left(t_{m}\right) & \leqslant\left(\lambda_{\max } \mathcal{M}_{m}^{2}\right) e^{\lambda\left(t_{m+1}-t_{m}\right)} M\|\xi\|_{\tau}^{2} e^{-\lambda\left(t_{b}-t_{0}\right)} \\
& <e^{-(\sigma+\lambda)\left(t_{m+1}-t_{m}\right)} e^{\lambda\left(t_{m+1}-t_{m}\right)} M\|\xi\|_{\tau}^{2} e^{-\lambda\left(t_{b}-t_{0}\right)} \\
& <M\|\xi\|_{\tau}^{2} e^{-\lambda\left(t_{b}-t_{0}\right)}=\mathbb{E} V\left(t_{b}\right) .
\end{aligned}
$$

So we can conclude from the definition of $t_{b}$ that there is $t_{a} \in\left[t_{m}, t_{b}\right)$, satisfying

$$
\mathbb{E} V\left(t_{a}\right)=\left(\lambda_{\max } \mathcal{M}_{m}^{2}\right) e^{\lambda\left(t_{m+1}-t_{m}\right)} M\|\xi\|_{\tau}^{2} e^{-\lambda\left(t_{b}-t_{0}\right)}
$$

Now, employing the methods in [28, (3.48)-(3.50)] results in

$$
\mathbb{E} V(t+s) \leqslant \gamma e^{\lambda \tau} \mathbb{E} V(t), \quad \forall t \in\left[t^{* *}, t^{*}\right], s \in[-\tau, 0],
$$


which together with $D^{+} \mathbb{E} V(t) \leqslant(\sigma-\lambda) V(t)$ and the condition $(\mathrm{H} 3)$ means

$$
\begin{aligned}
\mathbb{E} V\left(t_{b}\right) & \leqslant \mathbb{E} V\left(t_{a}\right) e^{(\sigma-\lambda)\left(t_{b}-t_{a}\right)} \\
= & \left(\lambda_{\max } \mathcal{M}_{m}^{2}\right) e^{\lambda\left(t_{m+1}-t_{m}\right)} M\|\xi\|_{\tau}^{2} e^{-\lambda\left(t_{b}-t_{0}\right)} e^{(\sigma-\lambda)\left(t_{b}-t_{a}\right)} \\
< & <e^{-(\sigma+\lambda)\left(t_{m+1}-t_{m}\right)} e^{\lambda\left(t_{m+1}-t_{m}\right)} M\|\xi\|_{\tau}^{2} e^{-\lambda\left(t_{b}-t_{0}\right)} e^{(\sigma-\lambda)\left(t_{b}-t_{a}\right)} \\
< & <\|\xi\|_{\tau}^{2} e^{-\lambda\left(t_{b}-t_{0}\right)}=\mathbb{E} V\left(t_{b}\right) .
\end{aligned}
$$

This contradiction verifies (11), and hence mathematical induction demonstrates the claim (2), which together with (4.25) means that the null solution of the impulsive system (4.20) is globally exponential with convergence rate $\frac{\lambda}{2}$.

\section{Numerical examples}

Example 5.1. In the system (2.1), set $\Omega=\left(-\frac{1}{2}, \frac{1}{2}\right) \times\left(-\frac{1}{2}, \frac{1}{2}\right) \times\left(-\frac{1}{2}, \frac{1}{2}\right)$, then $\lambda_{1} \geqslant 3$ ([11, Remark 14]). Set $\theta_{1}=\frac{1}{3}, \theta_{2}=\frac{1}{5}$, then the condition (H1) is satisfied. Assume, in addition,

$$
D=\left(\begin{array}{cc}
0.6 & 0 \\
0 & 0.5
\end{array}\right), B=\left(\begin{array}{cc}
1.5 & 0 \\
0 & 1.2
\end{array}\right)
$$

then $b_{i}<d_{i} \lambda_{1}$ and $0<\theta_{i}<1, \forall i=1,2$. Theorem 3.1 tells that the system (2.1) possesses at least three stationary solutions $(0,0),\left(u_{1 *}(x), 0\right)$ and $\left(0, u_{2 *}(x)\right)$, where $u_{i *}(x) \neq 0, \forall i=1,2$. Moreover, Theorem 3.2 yields that the zero solution $(0,0)$ of the system (2.1) is locally asymptotically stable.

Example 5.2. Set $S=\{1,2\}$, and $\gamma_{11}=-0.5, \gamma_{12}=0.5, \gamma_{21}=0.3, \gamma_{22}=-0.3, \Omega=[0,1] \times[0,1]$, and hence $\lambda_{1}=19.7392([11$, Remark 13] ). Let $\tau=1.5, \tau_{*}=0.85$ Set

$$
D=\left(\begin{array}{cc}
0.4 & 0 \\
0 & 0.35
\end{array}\right), B=\left(\begin{array}{cc}
1.5 & 0 \\
0 & 1.2
\end{array}\right), A=\left(\begin{array}{cc}
0.6 & 0 \\
0 & 0.55
\end{array}\right) \text {, }
$$

and $a_{12}=0.56, a_{21}=0.53, M_{1}=1=M_{2}, \theta_{1}=0.1, \theta_{2}=0.2$. Direct computation yields that (4.8) holds, which together with Theorem 4.1 implies that the null solution the the null solution is the unique stationary solution of the ecosystem (4.7) under the restrictive condition (4.1).

Moreover, applying computer Matlab LMI toolbox to (4.10) results in the feasible data:

$$
\begin{aligned}
P_{1}=\left(\begin{array}{cc}
1.0017 & 0 \\
0 & 1.0015
\end{array}\right), P_{2}=\left(\begin{array}{cc}
0.9987 & 0 \\
0 & 1.0003
\end{array}\right), Q_{1}=\left(\begin{array}{cc}
1.1177 & 0 \\
0 & 0.9996
\end{array}\right), Q_{2}=\left(\begin{array}{cc}
0.9993 & 0 \\
0 & 1.139
\end{array}\right), \\
W=\left(\begin{array}{cc}
1.1177 & 0 \\
0 & 0.9996
\end{array}\right), Q_{2}=\left(\begin{array}{cc}
0.9993 & 0 \\
0 & 1.1033
\end{array}\right) .
\end{aligned}
$$

Then Theorem 4.1 yields that the null solution of the ecosystem (4.7) is globally exponentially stable.

\section{Conclusions}

Gilpin-Ayala competition model with Dirichlet zero-boundary condition simulates well a class of actual ecological situation, but it brings out many difficulties on dynamical analysis of RDGACM. In this paper, the author employs mountain pass lemma, 
linear approximation principle, variational methods and free weight matrix technique to obtain the local stability and global stability of the zero solution of RDGACM. Theorems and numerical examples illuminate the effectiveness of the proposed methods. That is, the local asymptotical stability criterion shows that some suitable value can make both of two population densities of pests tend to zero eventually. Moreover, if the conditions of global exponential stability criterion (Theorem 4.1) are satisfied, no matter what the initial value is, both of two population densities must eventually tend to zero. The obtained Theorems and numerical examples illuminate that improving the diffusion of bacteria or pests is conducive to the elimination of pests or bacteria. For example, more ventilation in the area where bacteria are located is conducive to preventing the multiplication of bacteria and ultimately eliminating them.

Acknowledgement. The author thank Prof. Xinsong Yang, Doctor Rongqiang Tang, Doctor Yulin Zhang and Prof. Xinggui Li for their valuable suggestions and comments. Next step, the author will invite them as coauthors to complete together the final version of this manuscript.

\section{References}

[1] Lotka A. Elements of physical biology. Williams and Wilkins, Baltimore, Md., 1924.

[2] Volterra Vito. Lecons sur la Theorie Mathematique de la Lutte pour La Vie. Gauthier-Villars, Paris, 1931.

[3] Nanako Shigesada, Kohkichi Kawasaki, Ei Teramoto. Spatial segregation of interacting species. Journal of Theoretical Biology, Volume 79, Issue 17 July 1979Pages 83-99

[4] Yuanyuan Liu, Youshan Tao. Dynamics in a parabolic-elliptic two-species population competition model with cross-diffusion for one species. Journal of Mathematical Analysis and Applications, 2017, 456(11), 1-15.

[5] Gilpin, M.E., Ayala, F.J., Global models of growth and competition. Proceedings of the National Academy of Sciences of the United States of America, 70 (1973) 3590-3593

[6] Michael E. Gilpin, Francisco J. Ayala. Schoener's model and Drosophila competition. Theoretical Population Biology,1976, 9(1), 12-14

[7] William R. Thomas,Mark J. Pomerantz,Michael E. Gilpin. Chaos, Asymmetric Growth and Group Selection for Dynamical Stability. Ecology, 1980, 61,1312-1320. DOI: 10.2307/1939039.

[8] Meixiang Chen, Xizhuang Xie. Bi-stability of two-species competition model with reaction diffusion. Jounral of Huaqiao University (Natural Science), 2020, 41(2), 268-271.

[9] Moore, Christopher M.,Catella, Samantha A.,Abbott, Karen C. Population dynamics of mutualism and intraspecific density dependence: How $\theta$-logistic density dependence affects mutualistic positive feedback. Ecological Modelling Volume 368, 24 January 2018, Pages 191197

[10] Ruofeng Rao. Positive Solution for the Dirichlet Zero-Boundary Value Problem (In Chinese). College Mathematics,2010,26(02):146-152.

[11] Ruofeng Rao. Stability Analysis of Nontrivial Stationary Solution and Constant Equilibrium Point of Reaction-Diffusion Neural Networks with Time Delays under Dirichlet Zero Boundary Value. Preprints 2020, 2020040277 (doi: 10.20944/preprints202004.0277.v6).

[12] Haim Brezis, Louis Nirenberg. Remarks on finding critical points. Communications on Pure \& Applied Mathematics, 44 (1991), $939-963$.

[13] Willem M. Minimax theorems. Berlin: Birkhauser, 1996.

[14] Ji Y, Chizeck HJ. Controllability, stabilizability, and continuous-time Markovian jump linear quadratic control. IEEE Trans Autom Control 1990;35:777-88.

[15] Ruofeng Rao, Shouming Zhong, Xiongrui Wang. Stochastic stability criteria with LMI conditions for Markovian jumping impulsive BAM neural networks with mode-dependent time-varying delays and nonlinear reaction-diffusion. Communications in Nonlinear Science and Numerical Simulation, Volume 19, Issue 1January 2014Pages 258-273

[16] Ruofeng Rao and Shouming Zhong. Impulsive control on delayed feedback chaotic financial system with Markovian jumping. Advances in Difference Equations, 2020 2020:50 
[17] Ruofeng Rao, Xiongrui Wang.Infinitely Many Solutions for the Resonant Quasi-linear Equation Without Landesman-Lazer Conditions(In Chinese). Acta Mathematica Scientia, Ser. A, 2012,32(04):744-752.

[18] Ruofeng Rao.On the Elliptic Equations With the First Eigenvalue,Involving the Critial Sobolev Exponents (In Chinese). Advances In Mathematics (China), 2004(06):703-711.

[19] Liao Xiaoxin. Theory, method and application of stability (In Chinese). Huazhong Science and Technology Press, China, 2004.

[20] Ruofeng Rao, Quanxin Zhu, Kaibo Shi. Input-to-State Stability for Impulsive Gilpin-Ayala Competition Model With Reaction Diffusion and Delayed Feedback. IEEE Access, 2020, 8, 222625-222634.

[21] Ruofeng Rao. Existence, Uniqueness and Input-To-State Stability of Ground-State Stationary Strong Solution of a Single-Species Model via Mountain Pass Lemma. Preprints 2020, 2020090286 (doi: 10.20944/preprints202009.0286.v1).

[22] R. Rao, and S. Zhong, "Impulsive control on delayed feedback chaotic financial system with Markovian jumping," Advances in Difference Equa- tions, 2020 (2020) :50.

[23] Ruofeng Rao. Delay-Dependent exponential stability for nonlinear reaction-diffusion uncertain Cohen-Grossberg neural networks with partially known transition rates via Hardy-Poincare inequality. Chinese Annals of Mathematics, Series B, (2014),35, 575-598.

[24] Man-jun Ma, Jia-jia Hu, Jun-jie Zhang, Ji-cheng Tao. A reaction-diffusion model with nonlinearity driven diffusion. Applied MathematicsA Journal of Chinese Universities, 28, (2013), 290-302 .

[25] Ruofeng Rao, Shouming Zhong, Zhilin Pu. Fixed point and p-stability of T-S fuzzy impulsive reaction-diffusion dynamic neural networks with distributed delay via Laplacian semigroup. Neurocomputing, Volume 335, 28 March 2019, Pages 170-184

[26] Rao, R. Stability and stabilization of ecosystem for epidemic virus transmission under neumann boundary value via impulse control. Preprints 2021, 2021020197 (doi: 10.20944/preprints202102.0197.v1).

[27] Zhi-Ming Zhang, Yong He, Min Wu, and Qing-Guo Wang. Exponential Synchronization of Neural Networks With Time-Varying Delays via Dynamic Intermittent Output Feedback Control. IEEE Transactions on Systems, Man, and Cybernetics: Systems, VOL. 49, NO. 3 , MARCH 2019, 612-622.

[28] Ruofeng Rao, Shouming Zhong. Impulsive control on delayed feedback chaotic financial system with Markovian jumping. Advances in Difference Equations, 2020, 2020:50, 